\title{
IMAGES ANIMÉES MUETTES ET POLYSENSORIALITÉ \\ LE CAS EXEMPLAIRE DE CHAPLIN ET JOUR DE PAIE
}

\author{
Jacques Polet ${ }^{1}$
}

Loin de consister en une matière univoque réduite à sa pure "visualité", l'image animée muette est, à travers sa seule iconicité même, susceptible de décliner un vaste paradigme recouvrant en fait les catégories des cinq sens.

Il semblerait même que, appelée à ne fonctionner qu'avec une substance unique, elle ait été - elle soit— amenée à solliciter et à déployer d'autant plus les ressources de l'imaginaire sensoriel.

La période muette de Charlie Chaplin participe remarquablement de cette logique. C'est sous cet angle spécifique que nous avons choisi de traiter une œuvre dont la richesse, on le sait, ouvre de multiples entrées. Nous nous appuierons à cet effet sur le cas exemplaire d'un des tout derniers courts métrages réalisés par l'acteur-cinéaste, Jour de paie $(1922)^{2}$, dans lequel Charlot occupe l'emploi d'un travailleur de chantier de construction, pourvu par ailleurs d'une épouse passablement revêche...

1 Professeur émérite au Département de communication de l'UCL et à l'Institut des Arts de Diffusion (IAD).

2 Jour de paie est aussi un peu un film fétiche au sein du département COMU, ce qui ne constitue pas la moindre raison de notre choix...

Recherches en communication, $\mathrm{n}^{\circ} 29$ (2008). 
Le personnage de Charlot est immergé dans la trame sensorielle du monde diégétique représenté, comme chaque individu l'est dans son environnement. En effet, à travers une communication des sens, les stimulations se mêlent et se répondent en synesthésie, l'expérience humaine d'un registre sensoriel débordant "spontanément vers tous les autres"1.

Toutefois, par abstraction analytique, nous isolerons méthodologiquement ici chacun des sens inventoriés, en mettant plus particulièrement en évidence la dominante à l'œuvre à l'intérieur de situations que le film actualise.

Sans entrer, pour toutes raisons de lieu et d'objet, dans les débats sur la hiérarchie des sens alimentés par l'observation et la réflexion d'anthropologues et de philosophes à travers les siècles ${ }^{2}$, nous proposons de suivre l'ordre suivant : l'odorat, le goût, le toucher, l'ouïe, la vue.

On pourrait justifier cette consécution par une échelle ascendante de légitimation — du sens le plus trivial au sens réputé le plus noble-, mais aussi par l'évolution historique consacrant progressivement le primat de la vue ; encore que, en tout état de cause, l'organisation sensorielle ne soit pas indépendante des déterminations sociales et culturelles, comme le montrent notamment les études de terrain menées par les anthropologues.

En ce qui nous concerne, cet ordre nous apparaît avant tout pratique, en fournissant le fil approprié pour nous conduire à travers le régime sensoriel qui sous-tend ces images muettes.

\section{L'odorat et la prépondérance de la sensation sur le sentiment}

La situation la plus forte —et le qualificatif ne sera pas usurpécorrespond au début de la scène de la pause-déjeuner.

La fille du contremaître est assise à un des niveaux de l'échafaudage du chantier et s'apprête à extraire divers éléments de son panierrepas. Grâce au monte-charge, Charlot entreprend de se hisser jusqu'à une hauteur de surplomb par rapport à la jeune femme, de qui il obtient

1 M. Merleau-Ponty, Phénoménologie de la perception, Paris, Gallimard, 1945, p.273.

2 Nous renvoyons notamment à D. Ackerman, Le livre des sens, Paris, Grasset, 1990 ; D. Le Breton, La saveur du monde, Paris, Métailié, 2006 ; M. Serres, Les cinq sens, Paris, Hachette, 1985. 


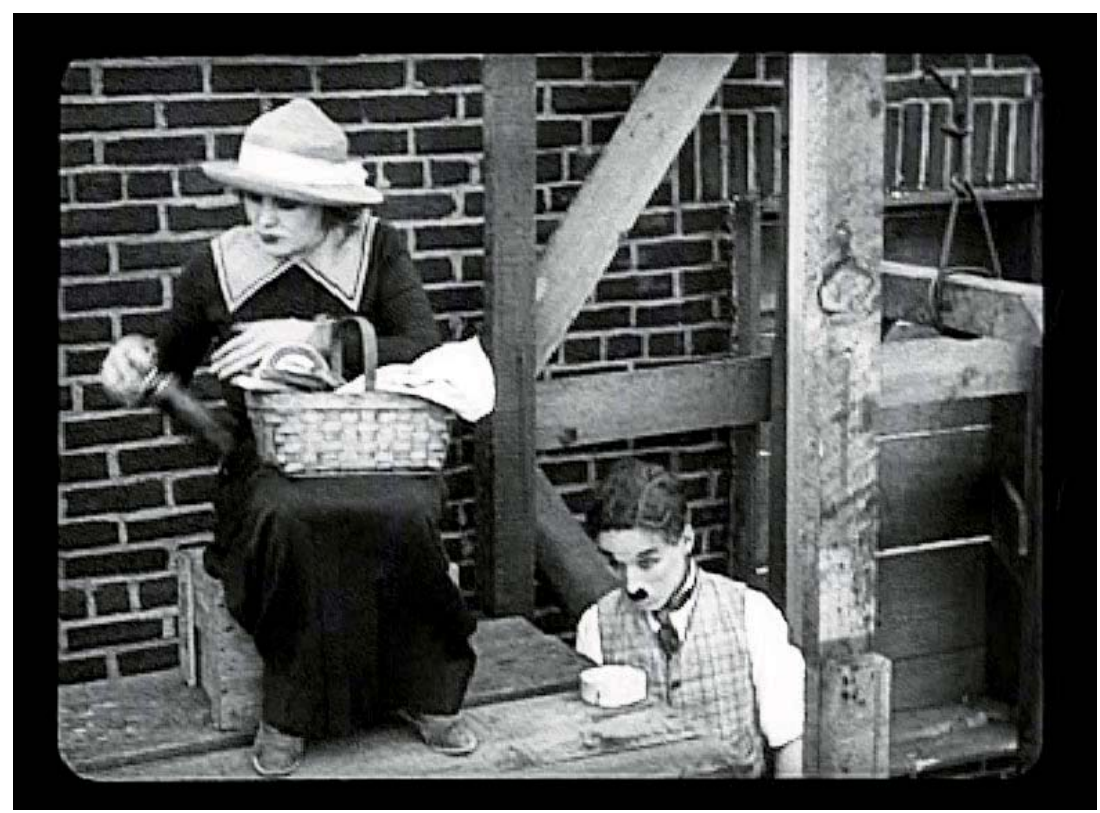

un sourire de sympathie. Renouvelant la démarche, il s'arrête cette fois en contre-bas d'elle. Le désir de Charlot est clairement affiché : on le voit, sur le monte-charge, inhaler on ne peut plus profondément la senteur qui émane pour lui de la jeune femme. Celle-ci découvre le manège ; confus, Charlot active le système de descente, mais, tenace, il se reporte au même point que précédemment et renouvelle la manifestation intense de sa volupté. Entre-temps, celle qui l'inspire tant a déposé auprès d'elle une boîte de fromage - camembert, en l'espèceet, progressivement, les sensations olfactives de l'homme semblent se brouiller. Mais c'est le regard de contrôle qu'il dirige vers le motif de ce subit désagrément qui ancre sa perception : la vue a relayé l'odorat pour fixer une sensation diffuse.

Ce qui se révèle singulièrement spectaculaire ici, c'est la conséquence qui en résulte sur l'attitude du personnage de Charlot : celle d'un retournement total. Alors que, précédemment, c'est l'étonnement de la jeune fille qui l'avait conduit à se retirer, ici c'est lui qui décide délibérément de manœuvrer l'appareil d'un geste de rejet souverain et impérieux : par le glissement olfactif se joue le renversement radical de l'attirance en répulsion. La sensation a tué le sentiment !

Alors que, comme le note David Le Breton, "l'appréciation des odeurs est un fait de circonstance" et que "le contexte où elles appa- 
raissent leur confère une valeur et un sens"1, ce qui nous est donné à éprouver ici, c'est la toute-puissance de l'olfaction en tant que telle, capable de se détacher et de s'abstraire d'une situation - le désir amoureux - susceptible pourtant de la déterminer par excellence et de recouvrir toute perception alternative.

En réalité, une contextualisation intervient néanmoins, mais elle ne relève pas de la même temporalité. Ce qui prédomine ici dans le champ odorant, c'est moins la relation immédiate au désir que le rapport médiat à la culture et à l'histoire. Le motif empirique du fromage qui est privilégié en l'occurrence n'est en effet pas innocent. Car ce qui ressurgit et interfère ici, c'est le vif discrédit dont ce produit a longtemps fait l'objet dans nos sociétés occidentales, en raison, notamment, de son origine, de sa forte exhalaison et de sa consistance liquide/solide.

Le film nous fait ainsi vérifier combien la perception sensorielle est effectivement médiée par des codes culturellement interprétés, et qu'en réalité la sensation fait sens.

A partir du motif du fromage, rappelé d'un film à l'autre de Chaplin, on pourrait même parler d'une forme de "mémoire olfactive"2 traversant son œuvre. On se souvient, en effet, que, dans Charlot soldat (1918), c'est de ce type de produit (un camembert bien fait) que le héros se sert comme d'une arme offensive, pour attaquer l'ennemi, après s'être luimême protégé d'un masque à gaz. Permanence, donc, de la connotation agressive et négative ${ }^{3}$.

1 D. Le Breton, op. cit., p. 256.

2 Ibid., pp.266-269. Diverses manifestations de la mémoire olfactive sont évoquées par l'auteur.

3 On pourrait s'amuser à imaginer une modification du statut culturel de cette gamme d'aliments quand on observe le succès récent et retentissant du long métrage Bienvenue chez les Ch'tis (Dany Boon, 2008) et qu'on apprend que les stocks de maroilles —ce fromage à senteur puissante typique du Nord de la France — se sont trouvés quasiment épuisés à un certain moment de l'année de la sortie du film (selon une information du journal télévisé de la RTBF du 9 avril 2008)... Même s'il serait audacieux d'entrevoir une mutation en l'espèce de la perception olfactive, on est en droit de subodorer, dans ce phénomène cinématographique de masse et son impact, un glissement, à tout le moins de conjoncture, de la lecture culturelle d'un produit générique. 


\section{Le goût et le manque}

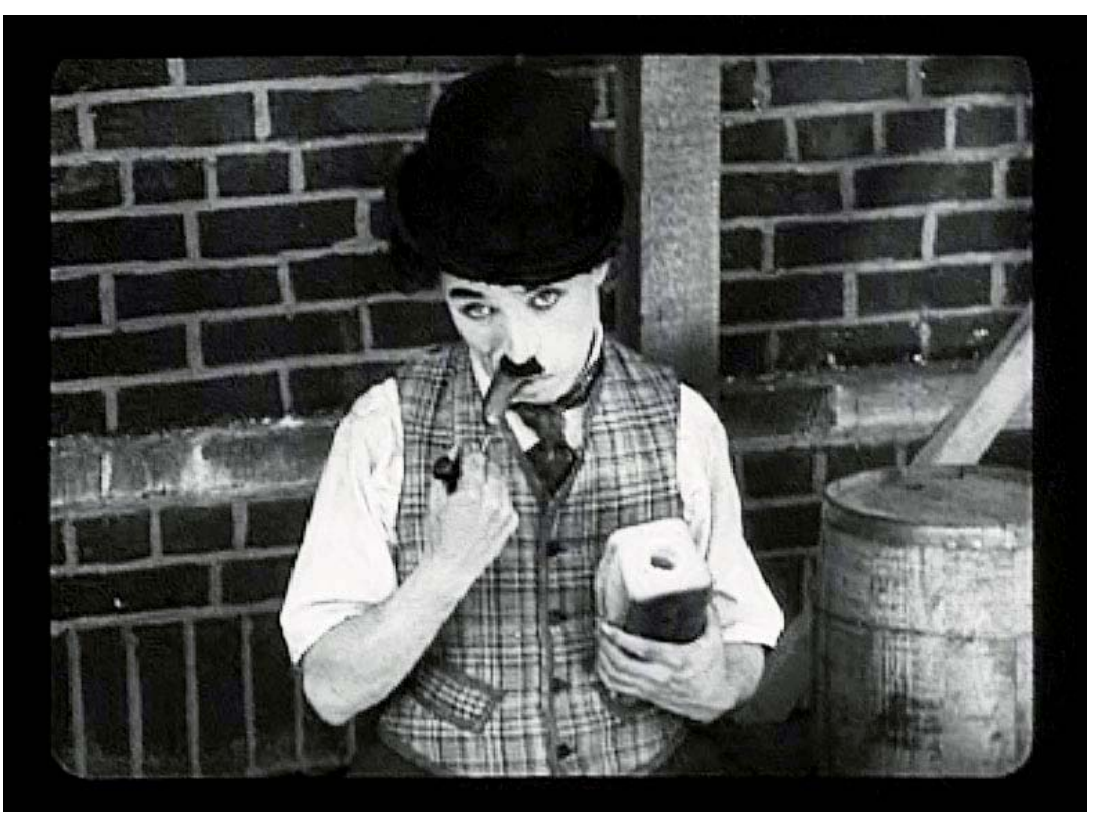

L'olfaction constitue l'antichambre du goût et elle l' "accompagne en permanence"'.

La séquence de la pause-repas se situe évidemment au cœur de l'acte de manger. Celui-ci est présenté comme problématique chez Charlot, en s'associant fondamentalement au manque.

Installé au milieu du dispositif de l'échafaudage — avec, au-dessus de lui, le contremaître et sa fille ; et, en dessous, ses deux compagnons de travail-, le protagoniste apparaît dépourvu de toute substance alimentaire, mais il tire parti de sa position intermédiaire pour glaner l'un ou l'autre éléments nutritifs, s'assurer un ersatz de menu inespéré et combler vaille que vaille le creux primitif.

Au départ, pourtant, les perspectives sont minimalistes. Charlot récupère un à un les petits fragments de pain qui ont glissé à travers les interstices de l'étage supérieur : la métaphore "recueillir les miettes du repas" est ici ramenée à la portée originelle de l'énoncé.

1 D. Le Breton, op.cit., p. 317. 
Mais c'est au fonctionnement aléatoire du monte-charge, dont il fait opportunément usage, que Charlot se constitue sa pitance. A la faveur des montées et descentes successives de la machine, il hérite tour à tour, involontairement, d'aliments de ses partenaires : saucisse, pain, banane.

La caméra capte ensuite de près et longuement l'acte de manducation, comme si elle souhaitait le donner d'abord à voir dans son fonctionnement physiologique — la bouche, "lieu manifeste de l'échange avec le monde", agent de l'intériorisation de l'extérieur ${ }^{1}$, avant que l'expression du personnage ne charge le biologique de signification, en témoignant du scepticisme gustatif du sujet.

Ce qui se joue avec l'élément solide trouve une correspondance dans la séquence finale avec l'élément liquide. Alors que Charlot a copieusement arrosé au bistrot sa paie du jour - le trop plein ici, après le trop vide initial—, on le voit, chez lui, avaler de l'eau, plusieurs fois, à petites gorgées. Bien sûr, il s'agit de signifier qu'il essaye de faire passer un funeste hoquet; mais, en amont, ce qui est proposé dans sa matérialité, c'est l'acte sensoriel même de la gutturation.

Enfin, normalement, comme le souligne David Le Breton, le repas "implique une commensalité, une mise en commun qui restitue les sensations à l'appréciation collective"'2. Le moins que l'on puisse dire est que cette structuration fait ici défaut. La nourriture ne donne pas lieu à partage ou à échange, mais elle se trouve redistribuée sur un mode fortuit entre des protagonistes séparés par les niveaux respectifs de la charpente. À l'horizontalité continue du dispositif convivial se substitue une verticalité fragmentée à la communication aléatoire.

Le langage filmique vient redoubler cette organisation spatiale du repas. En effet, la séquence ne comporte pas de plan d'ensemble et elle est fortement découpée, à l'image même de la dissociation des personnages.

A côté de l'eau ingérée par le héros —que nous avons évoquée précédemment-, la scène finale comporte une autre donnée alimentaire : le saucisson, que Charlot dépose sur la table mais que le chat, matois, lui dérobe subrepticement, lui ôtant son dernier support de gustation et le replaçant devant l'assiette vide. Autrement dit, face à son manque originel.

1 Ibid., p. 416.

2 Ibid., p. 378. C'est nous qui soulignons. 


\section{Le toucher et ses systèmes oppositionnels}

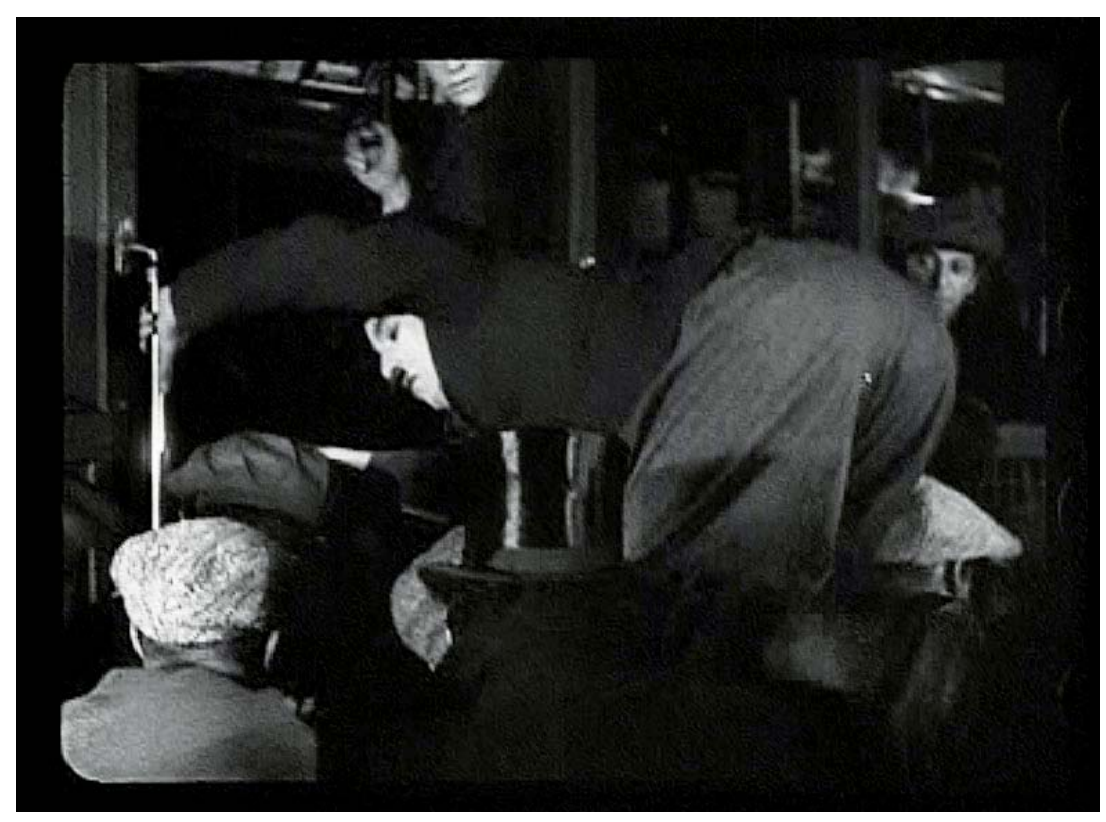

Le rapport à la nourriture met ici également en évidence le sens tactile.

Le passage clé à cet égard est celui où Charlot, par un hasard bienvenu, se voit disposer d'un pain qu'il palpe en tous sens, en éprouve la consistance rassie, creuse à l' aide d'une chignole, y introduisant ensuite une souple saucisse, qu'il enfonce à l'aide d'un marteau, pour la retirer en fin de compte avec un tire-bouchon et la porter à la bouche au bout de celui-ci. Il y a là une étonnante chaîne des touchers à travers laquelle fonctionne l'opposition du dur et du mou. Au point où, pour le premier pôle, la résistance du pain et la fine poussière qui s'en répand sont telles, que l'aliment semble revêtir la phénoménalité ... d'une brique, prolongeant ainsi, par le jeu de l'induction sémantique "dur comme une brique" parfois associée effectivement au pain, la métaphore des "miettes du repas" dégagée plus haut.

L'idée du contact physique du corps humain avec une matière quelconque est manifestée de façon aiguë au début précisément de la séquence de la pause-déjeuner, par le sursaut du protagoniste, debout sur les planches d'un palier de l'échafaudage : au geste qui suit, on comprend qu'il vient d'être piqué par la pointe d'un clou, lequel a 
traversé la semelle d'une de ses chaussures. $\mathrm{Si}$, comme le remarque Diane Ackerman, "une piqûre n'est sans doute pas ce qui fait le plus mal, mais c'est ce qui fait mal le plus vite"1, on saisit mieux le choix du motif opéré ici par Chaplin afin de visibiliser de la façon la plus spécifique la réaction des récepteurs nerveux à une stimulation sensorielle produite par le toucher.

Amorcé ainsi sur le registre de la douleur, un autre paradigme tactile se propose : celui du chaud/froid, recouvrant, d'une part, la sensation limite du brûlant stimulée par le tisonnier qui passe de main en main et touche violemment le bas d'un corps, et, d'autre part, le froid associé à l'eau du bain inattendu dans lequel Charlot s'immerge au petit matin, en cherchant une couche alternative dans la baignoire sur un amas de linge qui dissimule en fait le liquide sous-jacent. Ce qui construit l'opposition supplémentaire du sec et du mouillée.

La main se révèle ici par excellence "l'instrument des instruments" que lui reconnaissait Aristote $^{2}$. C'est une capacité exceptionnelle de préhension qui est donnée à voir, attestée de manière emblématique par la fameuse scène du lancer des briques où Chaplin, sur son promontoire, aux prises avec ces rudes objets que ses collègues lui transmettent, manifeste une adresse et une légèreté aériennes telles, que sa saisie infaillible en arrive à s'assimiler à de l'effleurement. Et c'est finalement tout le corps (dos, creux des genoux, menton) qui se trouve engagé dans l'exaltation de cette tactilité dont la souplesse infinie relève de la grâce.

On peut voir là un des volets d'un dernier système oppositionnel qu'on pointera encore. A cette grâce toute chorégraphique vient faire contraste la pesanteur du corps à corps impitoyable auquel Charlot se livre lorsqu'il monte désespérément à l'assaut du dernier tram bondé : il le fait au prix d'un toucher généralisé, désordonné, grouillant, investissant sans ménagement toute la matière du corps - ce qui n'empêche pas le héros, "transporté" bien malgré lui depuis l'arrière du convoi, de se faire éjecter par l'avant de celui-ci...

1 D. Ackerman, op. cit., p. 137.

2 Cité par D. Le Breton, entrée sur le "Toucher", in M. Marzano (dir.), Dictionnaire du corps, Paris, Presses Universitaires de France, 2007, p. 936. 


\section{L'ouïe et la stimulation de l'inventivité visuelle}

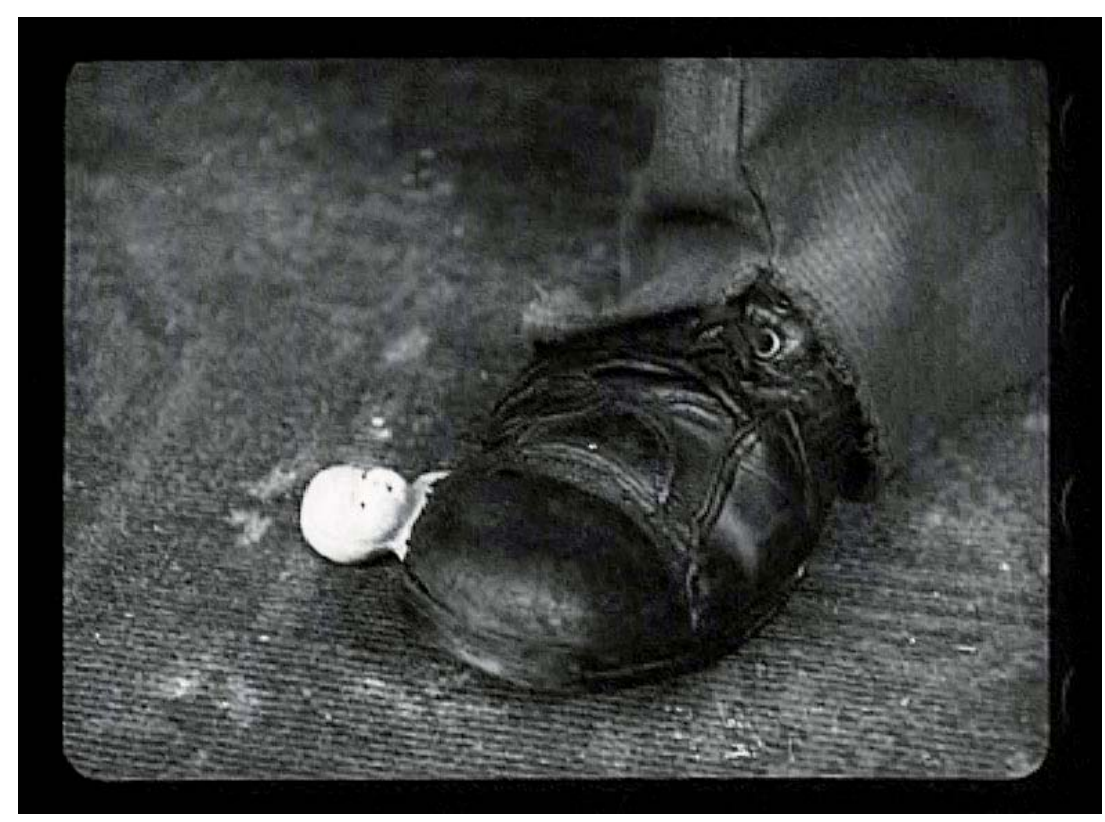

Les images animées muettes peuvent aussi rendre compte de sensations sonores, ce dont Jour de paie témoigne remarquablement, notamment par le jeu des mimiques surexpressives qui font "entendre" en l'espèce la chanson braillarde des compagnons fortement alcoolisés.

Mais la visualisation du son saute particulièrement aux yeux dans le dernier segment, qui couvre le retour tardif de Charlot au domicile.

Il s'agit pour lui d'éviter à tout prix de provoquer le réveil et le courroux redouté de son épouse : ses manifestations corporelles traduisent un luxe de précautions ambulatoires. Sur le plan iconique, il importe ici de sursignifier les effets de silence, pour mieux discriminer celui-ci de la mutité naturelle des images.

Mais la prudence affichée par le protagoniste ne réussit pas à conjurer les bruits intempestifs : la poupée de caoutchouc couine sous le pied, les souliers crissent sur le sol, Charlot veillant immédiatement à les graisser.

Comment, cette fois, accréditer la sensation sonore? A l'époque de la réalisation du film, où des cinéastes, notamment de la tendance burlesque, sensibles à la dimension attractionnelle du profilmique, sont encore marqués par le mode de représentation primitif —impli- 
quant particulièrement la caméra fixe, frontale, horizontale, à distance constante des motifs filmés-, il est intéressant d'observer l'usage, en l'occurrence, de gros plans et en plongée (sur la poupée, sur les souliers) pour matérialiser l'impression sonore.

A travers l'histoire du cinéma, il y a là, ponctuellement, une manifestation sémiologiquement exemplaire du développement d'un langage sous la pression des besoins d'un autre système de signes : dans ces circonstances, des contraintes sonores ont souvent poussé le langage visuel à explorer de nouvelles possibilités.

La séquence finale, riche décidément sur le terrain du son, comporte d'autres représentations intéressantes.

On remarque notamment, à l'opposé de la simulation du silence observée plus haut, le recours à la tactique de la feintise sonore, par laquelle, en ouvrant puis en fermant la porte d'entrée tout en restant à l'intérieur de l'habitation, Charlot compte bien faire accroire à son épouse qu'il a quitté le domicile pour se rendre à son travail.

Enfin, et surtout, on est fasciné par l'usage vertigineux qui est fait du motif du réveil-matin, que l'on peut mettre d'ailleurs en résonance — c'est le cas de le dire— avec celui du sifflet actionné par le contremaître du chantier.

Alors que, dans la chambre à coucher, Charlot entreprend de se déshabiller près du lit où son épouse est endormie, armée d'un rouleau de pâtisserie qui affiche clairement ses intentions, voici que le réveil sonne bien malencontreusement pour lui. (On notera le procédé chargé de visualiser la sonnerie et qui tient en un tremblement de l'objet, ce qui ne peut manquer de faire penser, pour des évocations analogues, au traitement, transposé, de certaines bulles dans l'univers de la bande dessinée.) Mais ce qui frappe singulièrement ici, c'est la réaction du héros à la sonnerie matinale fatidique marquant 5h25. Dans l'instant, alors donc qu'il a commencé à ôter ses vêtements, il fait mine, par un extraordinaire retournement, d'achever en fait de se vêtir : du coup, il n'est plus scandaleusement en retard -il est merveilleusement en avance ! La vitesse de son adaptation est telle qu'elle semble relever d'un pur réflexe face à un stimulus sonore.

Ce cas de figure peut être rapproché de celui dans lequel on voit le contremaître siffler l'interruption du travail avant la pause-repas. Sur-le-champ, Charlot, qui s'est hyperactivé en manipulant les briques, lâche celle qu'il tient à ce moment précis, et la voilà qui tombe sur la tête de son chef ! Ici encore, son geste relève de la réaction réflexe au facteur sonore. Mais il va plus loin en l'espèce : c'est dans le même 
mouvement que, réagissant au signal, Charlot tout à la fois obtempère à son supérieur et l'agresse. Objectivement, c'est sa soumission même qui permet sa violence, confondues toutes deux dans une conduite qui en vient à signifier une chose et son contraire.

\section{La vue et la valorisation de la position du spectateur}

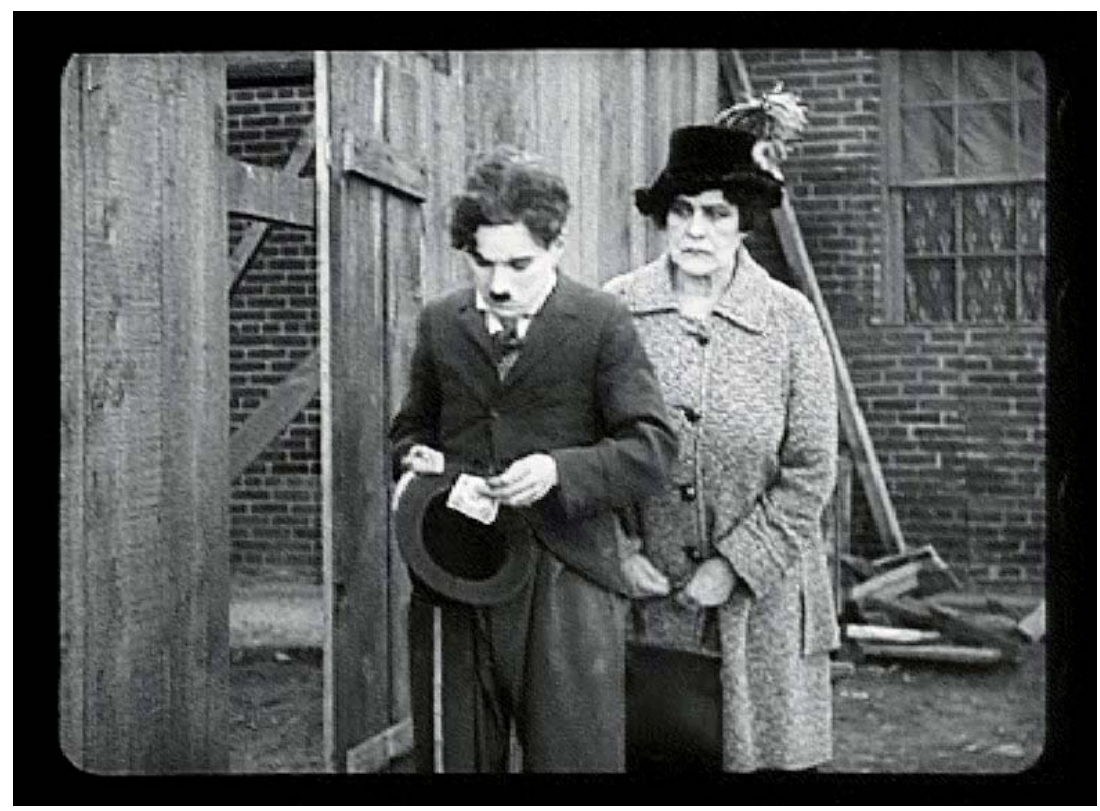

On le constate, ces images animées muettes excellent à faire voir des sensations appartenant aux divers régimes examinés jusqu'ici : l'odorat, le goût, le toucher, l'ouïe.

Curieusement, c'est dès lors le statut de la vue elle-même qui peut s'en trouver problématisé. Puisqu'une représentation purement iconique destinée par nature au "seul" regard réussit à solliciter en réalité autant de sens qui lui sont en principe" étrangers", quelle place peut-elle bien assigner à la vue qui lui est en quelque sorte consubstantielle, naturelle?

Il nous semble que c'est en adoptant une stratégie du point de vue. 
Nous l'expliciterons en nous référant à la typologie de l'ocularisation proposée par François Jost ${ }^{1}$.

Au-delà des images diverses relevant du type courant de l'ocularisation zéro, qui montrent des actions sans les attribuer au regard d'une instance intradiégétique, ce qui impressionne ici c'est la récurrence des ocularisations spectatorielles : autrement dit, de ces images filmées spécifiquement du point de vue du spectateur et qui lui octroient un avantage visuel par rapport aux personnages. Quelques exemples, parmi les plus significatifs :

- Le spectateur voit ce que Charlot, à l'extrême bord de l'échafaudage, ne voit pas : tournant le dos au monte-charge et en raison des mouvements de celui-ci, il est à plusieurs reprises menacé par le vide, mais il l'ignore 2 .

- Après la réception de sa paie, Charlot, qui entend bien la soustraire à son épouse dominatrice, en dissimule une partie à l'intérieur de son chapeau, mais il ne s'aperçoit pas que, à l'arrière, sa femme le file et se rend compte de son astuce; il ne la voit pas, elle le voit, et nous, en position frontale, nous voyons parfaitement les situations respectives.

- A la sortie de l'estaminet, une série d'images mettent à l'avant-plan le motif de la plaque d'égout. Contemplant ses compagnons de beuverie, Charlot s'appuie sur sa canne posée derrière lui. Il fait dos au spectateur qui est, quant à lui, en première loge, face à la grille sur laquelle, sans s'en douter, le héros déplace à plusieurs reprises sa badine, qui évite providentiellement de glisser dans les interstices : nous voyons le risque que le personnage ne perçoit pas, avant que la chute ne finisse par se produire.

1 A. Gaudreault et F. Jost, Le récit cinématographique, Paris, Nathan, 1990. Dans le chapitre 6 consacré au "Point de vue" (p.127-144), Jost distingue théoriquement les aspects cognitif (focalisation), visuel (ocularisation) et auditif (auricularisation). C'est de l'aspect proprement visuel que nous nous préoccupons ici, par la nature même de la présente approche.

2 Si Charlot résiste à la menace du vide, quelle que soit au demeurant son habileté, c'est précisément parce qu'il n'a pas conscience du danger, ce que l'on peut se représenter comme un corollaire du cas de figure du penseur de Pascal, que celui-ci suppose dans une situation physiquement délicate : "Le plus grand philosophe du monde, sur une planche plus large qu'il ne faut, s'il y a au-dessous un précipice, quoique sa raison le convainque de sa sûreté, son imagination prévaudra. Plusieurs n'en sauraient soutenir la pensée sans palir et suer". Voir Pascal, Pensées, Section II, Paris, Nelson, 1943, p. 82-83. 
- Dans la séquence finale du retour au domicile, Chaplin offre au spectateur, dès le début du montage, un plan montrant l'impérieuse compagne endormie, mais munie du rouleau de pâtisserie lourd de menace. Mis ainsi en évidence - comme on le dit en algèbre d'un nombre x placé devant un ensemble situé entre parenthèses-, ce plan détermine pour le spectateur tout l'enchaînement subséquent et il l'avertit en priorité du châtiment qui guette le héros.

Par ces privilèges visuels, le spectateur peut se donner ça et là l'illusion flatteuse d'une supériorité sur le personnage ${ }^{1}$, surtout quand celui-ci, comme c'est le cas de Charlot/Chaplin, se montre en mesure de faire preuve à ce point d'un rapport habile et inventif au monde et aux objets : pour tout dire, se révèle capable de telles performances.

Il est significatif à ce propos que la dernière modalité de l'ocularisation fasse ici défaut : l'ocularisation interne, qui suppose des images prises du point de vue propre au personnage, qu'il soit assumé directement par la caméra (ocularisation interne primaire) ou induit par le montage (ocularisation interne secondaire). Un plan est particulièrement révélateur à cet égard : celui qui cadre la palissade du chantier percé d'un trou, une sorte d'œilleton contre lequel Charlot pose son visage. On s'attend dès lors logiquement à la configuration regardant/ regardé, mais le second pôle n'est pas donné à voir, comme pour signifier — quasiment en creux et par l'absurde- le refus de l'instance filmante d'entrer dans la subjectivité du personnage. Celui-ci, ainsi que tout le reste, ne constitue en vérité qu'une donnée objective d'un spectacle susceptible de composer un vaste répertoire sensoriel reposant sur la forte sollicitation du regard et de l'expérience du spectateur.

1 Abordant sur le plan théorique la question du comique proprement dit, Freud a problématisé et relativisé le rôle qu'exercerait, sous cet angle spécifique du plaisir, le "sentiment de supériorité" du moi propre à l'égard d'autrui. Voir S. Freud, Le mot d'esprit et ses rapports avec l'inconscient, Paris, Gallimard, Idées, 1930, p. 314332. 


\section{Conclusion}

Construit et unifié autour du travail du corps de Chaplin, c'est tout un monde de sensations qui surgit des images cinématographiques, à la manière dont, comme le rapporte Merleau-Ponty, Cézanne soutenait qu' "un tableau contient en lui-même jusqu'à l'odeur du paysage", voulant dire par là, suivant l'auteur de la Phénoménologie de la perception, que "l'arrangement de la couleur sur la chose (...) signifie par luimême toutes les réponses qu'elle donnerait à l'interrogation des autres sens"1.

En manifestant une telle puissance d'évocation polysensorielle ${ }^{2}$, singulièrement exacerbée par la nature même de la démarche burlesque, des images animées muettes sont sans doute susceptibles de conduire le spectateur à faire retour sur ses propres aptitudes perceptives et à éprouver avec une plus claire conscience ce que, dans Les cinq sens, Michel Serres appelle "le premier cogito, plus enfoui quoique mieux affiché que le cogito de qui pense"3.

1 M. Merleau-Ponty, op.cit, Paris, Gallimard, 1945, p. 374.

2 Lepouvoir singulier de suggestion sensorielle dont les images animées muettes peuvent être dotées pose avec une particulière acuité la question de leur accompagnement sonore, lequel a été coutumier au cinéma depuis ses débuts dans les espaces de projection-réception. Voir notamment à ce sujet P. Bertolini et J. Polet, "Boniments, explications et autres bruits de scène. Les accompagnements de spectacles cinématographiques muets en Belgique", in Le bonimenteur de vues animées, Iris $\mathrm{n}^{\circ} 12$, automne 1996, p.145-160, et J. Polet, "Early Cinematographic Spectacles. The Role of Sound Accompaniment in the Reception of Moving Pictures", in R. Abel and R. Altman (eds.), The Sound of Early Cinema, Indiana University Press, 2001, p. 192-197. On peut se demander comment choisir le "ton juste", à travers la gamme des possibilités offertes entre la redondance et le contrepoint. Dans cet ordre d'idées, $\mathrm{Ph}$. Marion, narratologue et pianiste accompagnateur de films muets, s'est interrogé sur le mode d'actualisation de la musicalité potentielle de ces images, à l'occasion d'une contribution sur "Confusion sensorielle et musicalité virtuelle. Représentation de l'ivresse au cinéma muet”, in Cinéma \& Cie, n7, Fall 2005, p. 133-141.

3 M. Serres, op. cit., p. 67. 